\title{
EXPONENTIAL DICHOTOMIES AND FREDHOLM OPERATORS
}

\author{
KENNETH J. PALMER
}

(Communicated by Kenneth R. Meyer)

\begin{abstract}
It is shown that if the operator $(L x)(t)=\dot{x}(t)-A(t) x(t)$ is semi-Fredholm, then the differential equation $\dot{x}=A(t) x$ has an exponential dichotomy on both $[0, \infty)$ and $(-\infty, 0]$. This gives a converse to an earlier result.
\end{abstract}

1. Introduction. In [4] it was shown that if the linear system

$$
\dot{x}=A(t) x
$$

has an exponential dichotomy on $[0, \infty)$ and $(-\infty, 0]$, then the operator

$$
L: B C^{1}(-\infty, \infty) \rightarrow B C(-\infty, \infty)
$$

(see below for notations) defined by

$$
(L x)(t)=\dot{x}(t)-A(t) x(t)
$$

is Fredholm. The object of this note is to prove a converse to this.

2. Statement of the theorem. We denote by $A(t)$ an $n \times n$ matrix-valued function, bounded and continuous on an interval $J$, and by $X(t)$ a fundamental matrix for system (1). System (1) is said to have an exponential dichotomy on $J$ if there is a projection $P\left(P^{2}=P\right)$ and constants $K \geq 1, \alpha>0$ such that

$$
\begin{gathered}
\left|X(t) P X^{-1}(s)\right| \leq K e^{-\alpha(t-s)} \quad(s \leq t), \\
\left|X(t)(I-P) X^{-1)}(s)\right| \leq K e^{-\alpha(s-t)} \quad(s \geq t) .
\end{gathered}
$$

We denote by $B C(J)$ the Banach space of bounded continuous functions $x: J \rightarrow$ $\mathbf{R}^{n}$ with the norm

$$
\|x\|=\sup _{t \in J}|x(t)|
$$

and by $B C^{1}(J)$ the Banach space of continuously differentiable functions $x: J \rightarrow \mathbf{R}^{n}$ bounded together with their derivatives, where in the latter space we use the norm

$$
\|x\|_{1}=\sup _{t \in J}|x(t)|+\sup _{t \in J}|\dot{x}(t)| \text {. }
$$

With system (1) we associate the bounded linear operator $L: B C^{1}(J) \rightarrow B C(J)$ defined by (2) and denote by $\mathcal{N}(L)$ and $R(L)$ its nullspace and range. In Lemma

Received by the editors July $31,1987$.

1980 Mathematics Subject Classification (1985 Revision). Primary 34A30, 34C11; Secondary 47A53, 47A55.

Key words and phrases. Linear system, Fredholm operator, exponential dichotomy.

This work was presented at the Joint Mathematics Meetings held in Atlanta, Georgia, on January 6-9, 1988. 
4.2 in Palmer [4] it was shown that if equation (1) has exponential dichotomies on both $[0, \infty)$ and $(-\infty, 0]$, then the operator $L$ is Fredholm. Moreover it was shown that a function $f \in B C((-\infty, \infty))$ is in $R(L)$ if and only if $\int_{-\infty}^{\infty} \psi^{*}(t) f(t) d t=0$ ( ${ }^{*}$ denotes transpose) for all bounded solutions $\psi(t)$ of the adjoint equation

$$
\dot{x}=-A^{*}(t) x ;
$$

also the index of $L$ is the difference between the ranks of the projections associated with the dichotomies on $[0, \infty)$ and $(-\infty, 0]$.

THEOREM. Let $A(t)$ be an $n \times n$ matrix-valued function, bonded and continuous on an interval $J$, where $J=(-\infty, \infty),[0, \infty)$ or $(-\infty, 0]$. Suppose the operator $L: B C^{1}(J) \rightarrow B C(J)$ defined by $(2)$ is semi-Fredholm. Then when $J$ is a half-line, system (1) has an exponential dichotomy on $J$ and when $J=(-\infty, \infty)$, (1) has exponential dichotomies on both $[0, \infty)$ and $(-\infty, 0]$.

REMARK 1. The nullspace $\mathcal{N}(L)$ of $L$ is always finite-dimensional and complemented. For let $P$ be a projection with range $\left\{\xi \in \mathbf{R}^{n}: \sup _{t \in J}\left|X(t) X^{-1}(0) \xi\right|<\infty\right\}$. Then $\mathcal{N}(L)$ is the set of functions $X(t) \xi$ with $\xi$ in the range of $P$ and hence $\mathscr{N}(L)$ is finite-dimensional. Also $Y=\left\{x \in B C^{1}(J): P x(0)=0\right\}$ is a complement to $\mathcal{N}(L)$. So the assumption that $L$ is semi-Fredholm (see Kato [2, p. 230]) means simply that the range $R(L)$ of $L$ is closed.

REMARK 2 . When $L$ is surjective the result is already known (cf. Massera and Schäffer [3], Coppel [1]).

REMARK 3. The theorem shows that when $L$ is semi-Fredholm it must be, in fact, Fredholm and even surjective in the half-line case.

Thanks are due to Andrew Coppel for his suggestions concerning the work in this paper.

3. Proof of the Theorem. To prove the theorem we need two lemmas.

LEMMA 1. Let $A(t)$ be an $n \times n$ matrix-valued function, bounded and continuous on an interval $J$, when $J=(-\infty, \infty),[0, \infty)$ or $(-\infty, 0]$. Let $f \in B C(J)$ be of compact support. Then if $J$ is a half-line, the equation

$$
\dot{x}=A(t) x+f(t)
$$

has a solution $x(t)$ of compact support. If $J=(-\infty, \infty)$ equation (4) has a solution $x(t)$ of compact support if and only if $\int_{-\infty}^{\infty} \psi^{*}(t) f(t) d t=0$ for all solutions $\psi(t)$ of the adjoint equation (3).

Proof. First let $J=[0, \infty)$. Then any solution $x(t)$ of equation (4) can be written as

$$
x(t)=X(t) X^{-1}(0)\left\{\xi+X(0) \int_{0}^{t} X^{-1}(s) f(s) d s\right\} .
$$

Since $f$ has compact suport, there exists $\tau \geq 0$ such that $f(t)=0$ for $t \geq \tau$. Then we see that for $t \geq \tau$,

$$
x(t)=X(t) X^{-1}(0)\left\{\xi+X(0) \cdot \int_{0}^{\infty} X^{-1}(s) f(s) d s\right\} .
$$

So $x(t)$ has compact support if and only if $\xi=-X(0) \int_{0}^{\infty} X^{-1}(s) f(s) d s$. This proves the lemma for $J=[0, \infty)$. The proof for $J=(-\infty, 0]$ is similar. 
Now let $J=(-\infty, \infty)$. Then any solution $x(t)$ of equation (4) has the form (5). $x(t)$ has compact support in $[0, \infty)$ if and only if $\xi=-X(0) \int_{0}^{\infty} X^{-1}(s) f(s) d s$ and compact support in $(-\infty, 0]$ if and only if $\xi=X(0) \int_{-\infty}^{0} X^{-1}(s) f(s) d s$. These two values are the same if and only if $\int_{-\infty}^{\infty} X^{-1}(s) f(s) d s=0$. Since the transposes of the rows of $X^{-1}(t)$ form a basis for the solution space of the adjoint equation (3), this last condition is equivalent to the condition that $\int_{-\infty}^{\infty} \psi^{*}(s) f(s) d s=0$ for all solutions $\psi(t)$ of the adjoint equation (3). This completes the proof of the lemma.

Now we take

$$
\mathscr{F}=C_{0}(J)=\{f \in B C(J): f(t) \rightarrow 0 \text { as }|t| \rightarrow \infty\} .
$$

This is a closed subspace of $B C(J)$ in which the functions of compact support are dense. Since $L$ is continuous, $\xi=L^{-1}(\mathscr{F})$ is also a closed subspace of $B C^{1}(J)$. Then we define $T: \mathscr{E} \rightarrow \mathscr{F}$ to be the restriction of $L$ to $\mathscr{E}$. In the following lemma, we characterize $\mathscr{N}\left(T^{*}\right)$, where $T^{*}: \mathscr{F}^{*} \rightarrow \mathscr{E}^{*}$ is the conjugate operator.

LEMMA 2. Let $A(t), J$ be as in Lemma 1 , and let $T: \mathscr{E} \rightarrow \mathscr{F}$ be as just defined. Then if $J$ is a half-line, $\mathcal{N}\left(T^{*}\right)=\{0\}$. If $J=(-\infty, \infty), \alpha \in \mathscr{N}\left(T^{*}\right)$ if and only if there is a solution $\psi(t)$ of the adjoint equation (3) with $\int_{-\infty}^{\infty}|\psi(t)| d t<\infty$ such that for all $f$ in $\mathscr{F}$

$$
\alpha(f)=\int_{-\infty}^{\infty} \psi^{*}(t) f(t) d t
$$

In particular, this means that $\operatorname{dim} \mathscr{N}\left(T^{*}\right)<\infty$.

ProOF. Let $J$ be a half-line and let $f \in B C(J)$ be of compact support. Then by Lemma $1 f$ is in $R(L)$ and hence in $R(T)$. So $f=T x$ for some $x \in \mathscr{E}$. Therefore, if $\alpha \in \mathscr{N}\left(T^{*}\right)$,

$$
\alpha(f)=\alpha(T x)=\left(T^{*} \alpha\right)(x)=0 .
$$

This holds for all functions $f$ in $\mathscr{F}$ of compact support; these functions are dense in $\mathscr{F}$ and $\alpha$ is continuous. So $\alpha=0$, as required.

Now let $J=(-\infty, \infty)$ and suppose $\alpha \in \mathcal{N}\left(T^{*}\right)$. The following argument was suggested by Shilov [5, p. 25]. Let $\phi(t)$ be a positive function of compact support with $\int_{-\infty}^{\infty} \phi(t) d t=1$. Let $f \in \mathscr{F}$ be of compact support and define

$$
\tilde{f}(t)=f(t)-\phi(t) X(t) \int_{-\infty}^{\infty} X^{-1}(s) f(s) d s .
$$

Then we see that $\tilde{f}(t)$ has compact support and $\int_{-\infty}^{\infty} X^{-1}(t) \tilde{f}(t) d t=0$. So by Lemma $1, \tilde{f}$ is in $R(L)$ and hence in $R(T)$. That is, $\tilde{f}=T x$ for some $x$ in $\mathscr{E}$. Then since $\alpha \in \mathscr{N}\left(T^{*}\right)$,

$$
\alpha(\tilde{f})=\alpha(T x)=\left(T^{*} \alpha\right)(x)=0 .
$$

Then it follows from (7), (8) and a simple calculation that $\alpha(f)=\int_{-\infty}^{\infty} \psi^{*}(t) f(t) d t$, where

$$
\psi(t)=\sum_{i=1}^{n} \alpha\left(\phi(\cdot) x_{i}(\cdot)\right) \psi_{i}(t)
$$

$x_{i}(t)$ being the $i$ th column of $X(t)$ and $\psi_{i}(t)$ the transpose of the $i$ th row of $X^{-1}(t)$. 
Certainly $\psi(t)$ is a solution of the adjoint equation (3) and, using (6), for all functions $f \in \mathscr{F}$ of compact support,

$$
\left|\int_{-\infty}^{\infty} \psi^{*}(t) f(t) d t\right|=|\alpha(f)| \leq\|\alpha\|\|f\| .
$$

It follows that $\int_{-\infty}^{\infty}|\psi(t)| d t \leq\|\alpha\|<\infty$. Then $\alpha$ and $f \rightarrow \int_{-\infty}^{\infty} \psi^{*}(t) f(t) d t$ are both bounded linear functionals defined on $\mathscr{F}$ and coinciding on the dense subset consisting of the functions of compact suport. So (6) holds for all $f \in \mathscr{F}$, as required.

Conversely, suppose $\psi(t)$ is a solution of the adjoint equation (3) satisfying $\int_{-\infty}^{\infty}|\psi(t)| d t<\infty$. Then

$$
\int_{-\infty}^{\infty}|\dot{\psi}(t)| d t=\int_{-\infty}^{\infty}\left|A^{*}(t) \psi(t)\right| d t \leq \sup _{-\infty<t<\infty}\left|A^{*}(t)\right| \int_{-\infty}^{\infty}|\psi(t)| d t<\infty
$$

so that $\psi(t)=\psi(0)+\int_{0}^{t} \dot{\psi}(s) d s$ has limits as $t \rightarrow \pm \infty$. Since $\int_{-\infty}^{\infty}|\psi(t)| d t<\infty$, these limits must both be zero. So $\psi(t) \rightarrow 0$ as $|t| \rightarrow \infty$.

Now $\alpha$ defined by (6) is certainly in $\mathscr{F}^{*}$. Moreover, if $x \in \mathscr{E}$,

$$
\begin{aligned}
\left(T^{*} \alpha\right)(x) & =\alpha(T x)=\int_{-\infty}^{\infty} \psi^{*}(t)[\dot{x}(t)-A(t) x(t)] d t \\
& =\left[\psi^{*}(t) x(t)\right]_{-\infty}^{\infty}-\int_{-\infty}^{\infty}\left[\dot{\psi}^{*}(t)+\psi^{*}(t) A(t)\right] x(t) d t \\
& =0
\end{aligned}
$$

PROOF OF THE THEOREM. We are supposing that $L$ is semi-Fredholm. So $R(L)$ is closed. Hence $R(T)$ is closed also. Then by Theorem 4.6- $\mathrm{C}$ in Taylor [6, p. 226],

$$
\{R(T)\}^{0}:=\left\{\alpha \in \mathscr{F}^{*}: \alpha(f)=0 \forall f \in R(T)\right\}=\mathscr{N}\left(T^{*}\right) .
$$

Suppose now $J$ is a half-line. Then by Lemma $2, \mathcal{N}\left(T^{*}\right)=\{0\}$. So by the Hahn-Banach theorem, $R(T)=\mathscr{F}$. That is, for all $f \in B C(J)$ with $f(t) \rightarrow 0$ as $t \rightarrow \pm \infty$ ( + when $J=[0, \infty),-$ when $J=(-\infty, 0])$, equation (4) has a solution bounded on $J$. Then it follows from Theorem 64.B in Massera and Schäffer [3, p. 189] or from the proof of Proposition 3 in Coppel [1, p. 22] that equation (1) has an exponential dichotomy on $J$.

Suppose now $J=(-\infty, \infty)$. It is an exercise to prove that since $\{R(T)\}^{0}=$ $\mathscr{N}\left(T^{*}\right)$ and $\operatorname{dim} \mathscr{N}\left(T^{*}\right)<\infty$, then $R(T)$ is saturated (Taylor [6, p. 225]). That is,

$$
R(T)={ }^{0}\left\{\mathscr{N}\left(T^{*}\right)\right\}:=\left\{f \in \mathscr{F}: \alpha(f)=0 \forall \alpha \in \mathscr{N}\left(T^{*}\right)\right\} .
$$

By Lemma 2 this means that $f \in R(T)$ if and only if $\int_{-\infty}^{\infty} \psi^{*}(t) f(t) d t=0$ for all solutions $\psi(t)$ of the adjoint equation (3) satisfying $\int_{-\infty}^{\infty}|\psi(t)| d t<\infty$.

Now let $\psi_{1}(t), \psi_{2}(t), \ldots, \psi_{m}(t)$ be a basis for the subspace of solutions $\psi(t)$ of equation (3) satisfying $\int_{-\infty}^{\infty}|\psi(t)| d t<\infty$. Let $f \in C_{0}([0, \infty))$. We seek $g \in$ $C_{0}((-\infty, 0])$ such that

$$
\int_{-\infty}^{0} \psi_{i}^{*}(t) g(t) d t=-\int_{0}^{\infty} \psi_{i}^{*}(t) f(t) d t \quad(i=1, \ldots, m)
$$




$$
g(0)=f(0)
$$

Such a $g$ certainly exists provided the bounded linar functionals on $C_{0}((-\infty, 0])$ defined by

$$
\begin{aligned}
& \alpha_{i}(g)=\int_{-\infty}^{0} \psi_{i}^{*}(t) g(t) d t \quad(i=1, \ldots, m), \\
& \beta_{j}(g)=g_{j}(0) \quad(j=1, \ldots, n),
\end{aligned}
$$

where $g_{j}(t)$ is the $j$ th component of $g(t)$, are linearly independent.

Suppose there are scalars $\gamma_{i}(i=1, \ldots, m), \delta_{j}(j=1, \ldots, n)$ such that $\sum_{k=1}^{m} \gamma_{i} \alpha_{i}$ $=\sum_{j=1}^{n} \delta_{j} \beta_{j}$. Then for all $g$ in $C_{0}((-\infty, 0])$,

$$
\int_{-\infty}^{\infty} \psi^{*}(t) g(t) d t=\sum_{j=1}^{n} \delta_{j} g_{j}(0),
$$

where $\psi(t)=\sum_{i=1}^{m} \gamma_{i} \psi_{i}(t)$. Suppose $\psi \neq 0$. Then we can choose $g$ so that $\psi^{*}(t) g(t)>0$ for all $t$ but $g(0)=0$. Then the left side of (11) would be positive but the right side zero. So $\psi=0$. Since the $\psi_{i}$ 's are linearly independent, it follows that $\gamma_{i}=0$ for all $i$. Then for all $g, \sum_{j=1}^{n} \delta_{j} g_{j}(0)=0$. For each $j$ we can choose $g$ so that $g_{j}(0)=1$ but $g_{k}(0)=0$ for $k \neq j$. So we conclude that $\delta_{j}=0$ for all $j$ also. Hence the linear functionals are linearly independent and so $g \in C_{0}((-\infty, 0])$ can be chosen so that (9) and (10) are satisfied.

Now we define $\tilde{f}(t)$ to be $f(t)$ for $t \geq 0$ and $g(t)$ for $t<0$. Then $\tilde{f} \in C_{0}((-\infty, \infty))$ and $\int_{-\infty}^{\infty} \psi_{i}^{*}(t) f(t) d t=0$ for $i=1, \ldots, m$. So $\tilde{f} \in R(T)$. This means that the equation $\dot{x}=A(t) x+\tilde{f}(t)$ has a solution bounded on $(-\infty, \infty)$. Restricting to $[0, \infty)$ we conclude that equation (4) has a solution bounded on $[0, \infty)$. This holds for all $f$ in $C_{0}([0, \infty))$ and so, by the result of Massera and Schäffer used earlier, it follows that equation (1) has an exponential dichotomy on $[0, \infty)$. A similar argument shows that it has an exponential dichotomy on $(-\infty, 0]$. So the proof of the theorem is complete.

4. The effect of perturbations. If $\tilde{L}$ is a small perturbation of a Fredholm operator $L$, then $\tilde{L}$ is also Fredholm, has the same index as $L$ and $\operatorname{dim} \mathscr{N}(\tilde{L}) \leq$ $\operatorname{dim} \mathscr{N}(L)$. We want to show that both the possibilities $\operatorname{dim} \mathscr{N}(\tilde{L})=\operatorname{dim} \mathscr{N}(L)$ and $\operatorname{dim} \mathscr{N}(\tilde{L})<\operatorname{dim} \mathscr{N}(L)$ do arise in the case of differential equations.

First we look at an abstract situation.

Proposition. Let $\mathscr{E}, \mathscr{F}$ be Banach spaces, L: $\mathscr{E} \rightarrow \mathscr{F}$ a Fredholm operator of index zero with $\operatorname{dim} \mathscr{N}(L)=1$ and let $N_{i}: \mathscr{E} \rightarrow \mathscr{F}(i=1,2)$ be bounded linear operators.

Suppose that not both $N_{1} \phi$ and $N_{2} \phi$ are in $R(L)$, where $\phi \in \mathscr{N}(L), \phi \neq 0$. Then there is a neighborhood $O$ of the origin in $\mathbf{R}^{2}$ and a curve passing through the origin such that when $\left(\mu_{1}, \mu_{2}\right) \in O, \operatorname{dim} \mathscr{N}\left(L+\mu_{1} N_{1}+\mu_{2} N_{2}\right)=1$ if $\left(\mu_{2}, \mu_{2}\right)$ is on the curve and $=0$ otherwise.

PROOF. The proof is a straightforward application of the Lyapunov-Schmidt method. 
Let $\alpha$ be a bounded linear functional on $\mathscr{E}$ such that $\alpha(\phi)=1$. Also let $\beta$ be a bounded linear functional on $\mathscr{F}$ such that $R(L)=\mathcal{N}(\beta)$ and let $\eta \in F$ satisfy $\beta(\eta)=1$. We seek those values of $\left(\mu_{1}, \mu_{2}\right)$ for which the equation

$$
\left(L+\mu_{1} N_{1}+\mu_{2} N_{2}\right) x=0
$$

has nonzero solutions $x \in \mathscr{E}$. We may write $x=\gamma \phi+w$ where $\gamma$ is real and $w \in \mathcal{N}(\alpha)$. Then we want to solve

$$
L w=-\left(\mu_{1} N_{1}+\mu_{2} N_{2}\right)(\gamma \phi+w)
$$

for $\gamma$ and $w$. Solution of this equation is equivalent to solution of the pair of equations

$$
\begin{gathered}
L w=-Q\left(\mu_{1} N_{1}+\mu_{2} N_{2}\right)(\gamma \phi+w), \\
\beta\left(\left(\mu_{1} N_{1}+\mu_{2} N_{2}\right)(\gamma \phi+w)\right)=0,
\end{gathered}
$$

where $Q$ is the projection on $\mathscr{F}$ defined by $Q(y)=y-\beta(y) \eta$. Note that $R(Q)=$ $R(L)$.

By the bounded inverse theorem, $L: \mathcal{N}(\alpha) \rightarrow R(L)$ is invertible. So when $\mu_{1}, \mu_{2}$ are small, the operator $K\left(\mu_{1}, \mu_{2}\right)=L+Q\left(\mu_{1} N_{1}+\mu_{2} N_{2}\right): \mathscr{N}(\alpha) \rightarrow R(L)$ is also invertible. So we can solve (12) for $w=\gamma w\left(\mu_{1}, \mu_{2}\right)$, where $w\left(\mu_{1}, \mu_{2}\right)=$ $-K\left(\mu_{1}, \mu_{2}\right)^{-1} Q\left(\mu_{1} N_{1}+\mu_{2} N_{2}\right)(\phi)$. Note that $w\left(\mu_{1}, \mu_{2}\right)$ is smooth with $w(0,0)=0$. Then we substitute $w=\gamma w\left(\mu_{1}, \mu_{2}\right)$ into equation (13) to get an equation

$$
\gamma h\left(\mu_{1}, \mu_{2}\right)=0
$$

where

$$
h\left(\mu_{1}, \mu_{2}\right)=\beta\left(\left(\mu_{1} N_{1}+\mu_{2} N_{2}\right)\left(\phi+w\left(\mu_{1}, \mu_{2}\right)\right) .\right.
$$

Now $x=\gamma\left(\phi+w\left(\mu_{1}, \mu_{2}\right)\right)$ where $w(0,0)=0$. So if $\mu_{1}, \mu_{2}$ are sufficiently small, $x \neq 0$ if and only if $\gamma \neq 0$. Thus $\mathcal{N}\left(L+\mu_{1} N_{1}+\mu_{2} N_{2}\right) \neq\{0\}$ if and only if $h\left(\mu_{1}, \mu_{2}\right)=0$.

Furthermore, $h(0,0)=0$ and $\partial h(0,0) / \partial \mu_{i}=\beta\left(N_{i} \phi\right)(i=1,2)$. Since $N_{i} \phi \notin$ $R(L)$ for some $i, \partial h(0,0) / \partial \mu_{i} \neq 0$ for some $i$. So by the implicit function theorem there is a neighborhood $O$ of the origin in $\mathbf{R}^{2}$ and a curve through the origin such that when $\left(\mu_{1}, \mu_{2}\right) \in O, h\left(\mu_{1}, \mu_{2}\right)=0$ if and only if $\left(\mu_{1}, \mu_{2}\right)$ lies on the curve. This completes the proof of the proposition.

EXAMPLE. Let $g(x)$ be a $C^{1}$ real function with $g(0)=0, g^{\prime}(0)<0$. This implies that the origin (in phase space) is a saddle point for the equation

$$
\ddot{x}+g(x)=0 .
$$

We also suppose the equation has a homoclinic solution; that is, a solution $\varsigma(t) \rightarrow 0$ as $|t| \rightarrow \infty$. Then stable manifold theory tells us that $\zeta(t)$ and $\dot{\zeta}(t) \rightarrow 0$ exponentially as $|t| \rightarrow \infty$.

We consider the variational equation

$$
\ddot{x}+g^{\prime}(\varsigma(t)) x=0
$$

and its perturbation

$$
\ddot{x}+\mu_{1} \dot{x}+\left[g^{\prime}(\zeta(t))+\mu_{2}\right] x=0 .
$$


As a system this has the form

$$
\dot{x}=\left[A(t)+\mu_{1} B_{1}+\mu_{2} B_{2}\right] x,
$$

where $x \in \mathbf{R}^{2}$ and

$$
A(t)=\left[\begin{array}{cc}
0 & 1 \\
-g^{\prime}(\varsigma(t)) & 0
\end{array}\right], \quad B_{1}=\left[\begin{array}{cc}
0 & 0 \\
0 & -1
\end{array}\right], \quad B_{2}=\left[\begin{array}{cc}
0 & 0 \\
-1 & 0
\end{array}\right] .
$$

The corresponding operator from $B C^{1}((-\infty, \infty))$ into $B C((-\infty, \infty))$ has the form $L+\mu_{1} N_{1}+\mu_{2} N_{2}$, where $L$ is defined by $(2)$ and $\left(N_{i} x\right)(t)=B_{i} x(t)(i=1,2)$.

Now system (1) with $A(t)$ as above has the property that $A(t) \rightarrow C=\left[\begin{array}{cc}0 & 1 \\ -g^{\prime}(0) & 0\end{array}\right]$ as $|t| \rightarrow \infty$. The eigenvalues of $C$ are $\pm \sqrt{-g^{\prime}(0)}$ and so $\dot{x}=C x$ has an exponential dichotomy on $(-\infty, \infty)$ with projection of rank 1 . It follows from the roughness theorem (Coppel [1, p. 34]) that system (1) has exponential dichotomies on intervals $[\tau, \infty)$ and $(-\infty,-\tau]$ for $\tau$ sufficiently large and hence on both half-lines by Coppel $[\mathbf{1}$, p. 13]. Also the projections involved have rank 1. Then it follows from Lemma 4.2 in Palmer [4] that the operator $L: B C^{1}((-\infty, \infty)) \rightarrow B C((-\infty, \infty))$ defined by

(2) is Fredholm of index zero. Next we see by inspection that

$$
\phi(t)=\left[\begin{array}{l}
\dot{\zeta}(t) \\
\ddot{\zeta}(t)
\end{array}\right]
$$

is a solution of equation (1) bounded on $(-\infty, \infty)$. Since the dimensions of the subspaces of solutions bounded on the half-lines are 1 , it follows that $\phi(t)$ is, up to a scalar multiple, the unique solution of (1) bounded on $(-\infty, \infty)$. Hence $\operatorname{dim} \mathscr{N}(L)=1$.

We also see by inspection that

$$
\psi(t)=\left[\begin{array}{c}
\ddot{\zeta}(t) \\
-\dot{\zeta}(t)
\end{array}\right]
$$

is a bounded solution of the adjoint equation (3). From the proof of Lemma 4.2 in Palmer [4], the subspaces of the bounded solutions (on $(-\infty, \infty)$ ) of equations (1) and (3) have the same dimension (in this case). So up to a scalar multiple, $\psi(t)$ is the unique bounded solution of (3). Moreover it follows from the same lemma that

$$
f(t)=\left[\begin{array}{l}
f_{1}(t) \\
f_{2}(t)
\end{array}\right]
$$

is in the range of $L$ if and only if

$$
\int_{-\infty}^{\infty} \psi^{*}(t) f(t) d t=\int_{-\infty}^{\infty}\left[\ddot{\zeta}(t) f_{1}(t)-\dot{\zeta}(t) f_{2}(t)\right] d t=0 .
$$

Now

$$
\begin{gathered}
\int_{-\infty}^{\infty} \psi^{*}(t)\left(N_{1} \phi\right)(t) d t=\int_{-\infty}^{\infty} \ddot{\zeta}(t) \dot{\zeta}(t) d t=0 \\
\int_{-\infty}^{\infty} \psi^{*}(t)\left(N_{2} \phi\right)(t) d t=\int_{-\infty}^{\infty} \dot{\zeta}(t)^{2} d t>0
\end{gathered}
$$

So $N_{2} \phi \notin R(L)$ and the Proposition implies there is a neighborhood $O$ of $(0,0)$ in $\mathbf{R}^{2}$ and a curve passing through the origin such that when $\left(\mu_{1}, \mu_{2}\right) \in O$, $\operatorname{dim} \mathscr{N}\left(L+\mu_{1} N_{1}+\mu_{2} N_{2}\right)=1$ if $\left(\mu_{1}, \mu_{2}\right)$ is on the curve and $=0$ otherwise. 


\section{REFERENCES}

1. W. A. Coppel, Dichotomies in stability theory, Lecture Notes in Math., vol. 629, SpringerVerlag, Berlin, 1978.

2. T. Kato, Perturbation theory of linear operators, Springer-Verlag, Berlin, 1976.

3. J. L. Massera and J. J. Schäffer, Linear differential equations and function spaces, Academic Press, New York, 1966.

4. K. J. Palmer, Exponential dichotomies and transversal homoclinic points, J. Differential Equations 55 (1984), 225-256.

5. G. E. Shilov, Generalized functions and partial differential equations, Gordon and Breach, New York, 1968.

6. A. E. Taylor, Introduction to functional analysis, Wiley, New York, 1958.

Department of Mathematics and Computer Science, University of Miami, Coral Gables, Florida 33124 